\title{
Generation of an intense cold-atom beam from a pyramidal magneto-optical trap: experiment and simulation
}

\author{
James M. Kohel, Jaime Ramirez-Serrano, Robert J. Thompson, and Lute Maleki \\ Jet Propulsion Laboratory, California Institute of Technology, 4800 Oak Grove Drive, Pasadena, California 91109- \\ 8099 \\ Joshua L. Bliss and Kenneth G. Libbrecht \\ Department of Physics, Norman Bridge Laboratory, California Institute of Technology, Pasadena, California 91125
}

Received July 24, 2002; revised manuscript received January 24, 2003

\begin{abstract}
An intense cold-atom beam source based on a modified pyramidal magneto-optical trap has been developed and characterized. We have produced a slow beam of cold cesium atoms with a continuous flux of 2.2 $\times 10^{9}$ atoms $/ \mathrm{s}$ at a mean velocity of $15 \mathrm{~m} / \mathrm{s}$ and with a divergence of $15 \mathrm{mrad}$. The corresponding radiant intensity is $1.2 \times 10^{13}$ atom s$~ s^{-1} \mathrm{sr}^{-1}$. We have characterized the performance of our beam source over a range of operating conditions, and the measured values for atom flux, mean velocity, and divergence are in good agreement with results from detailed Monte Carlo numerical simulations. (c) 2003 Optical Society of America OCIS codes: $140.3320,020.7010$.
\end{abstract}

\section{INTRODUCTION}

Techniques to generate cold-atom beams are of great interest in a variety of applications, from atomic frequency standards and precision atomic resonance experiments to atom optics and studies of Bose-Einstein condensation. Cold-atom beams have been produced by slowing thermal atomic beams with the Zeeman-slowing technique ${ }^{1}$ or chirped lasers ${ }^{2}$ or with laser-cooling techniques to extract a slow atomic beam from the background gas in a lowpressure vapor cell. These laser-cooled slow-atom sources, often referred to as "atom funnels," include twodimensional magneto-optical traps ${ }^{3-7}$ as well as a variation $^{8}$ of the three-dimensional (3D) vapor cell magneto-optical trap (MOT).

In the atom funnel derived from a 3D-MOT, a cold-atom beam is formed in an extraction column defined by a small aperture in the center of one of the retro-optics. The aperture produces an imbalance in radiation pressure in a narrow column along one axis. Atoms at the center of the trap are accelerated toward the aperture by this imbalance and thus form a slow $(<30 \mathrm{~m} / \mathrm{s})$ beam of cold atoms. The divergence of the extracted beam is quite small, typically $40 \mathrm{mrad}$ or less, and the brightness can be more than an order of magnitude greater than that of slowed thermal beam sources. The slow mean velocity of atoms in this beam is well matched to the capture velocity of typical magneto-optic traps, allowing efficient loading of a second MOT from this beam. With proper design, a very high transfer efficiency of atoms through the exit aperture can be obtained while maintaining a very low conductance between the source region and an adjacent chamber. This facilitates the use of differential pumping to obtain ultrahigh-vacuum pressures in the ad- jacent chamber. These features make this laser-cooled atomic beam source particularly well suited to cold-atom experiments requiring very low background pressures and fast trap-loading rates, such as studies of BoseEinstein condensates.

For trap-loading applications, a useful figure of merit for the performance of a slow-atom beam is the radiant intensity, defined as the rate of atoms emitted per unit of solid angle. ${ }^{9}$ The combination of high flux and low divergence for the slow beam allows the rapid loading of an ultrahigh-vacuum MOT located many tens of centimeters downstream from the source region. The 3D-MOT-based source in the present work employs a pyramidal trap geometry, ${ }^{10}$ similar to previous work, ${ }^{11-13}$ but in which the pyramidal mirrors are truncated before the apex. This allows the incorporation of a separate retro-optic containing the exit aperture. The reflection from this retro-optic provides a well-defined extraction column along the longitudinal axis of the trap for the cold-atom beam. A single large-diameter (15-cm) laser beam is used to obtain high capture rates of atoms from the background vapor. This simple design provides a robust atomic beam with high radiant intensity.

In this report, we describe the characterization of this cold-atom beam source over a range of operating conditions. We also present a detailed Monte Carlo numerical model of this laser-cooled system, and find good agreement with measured values for the atomic beam flux, velocity, and divergence.

\section{EXPERIMENTAL APPARATUS}

The pyramidal trap is realized by four gold-coated glass mirrors, chosen for their high optical quality, which form 
the four sectors of the pyramidal mirror assembly plus a retro-optic consisting of a $\lambda / 4$ plate with a high-reflectance gold coating on the second surface and a 1-mm laserdrilled aperture at the center. The four mirrors are assembled to form a $90^{\circ}$ (included angle) pyramid that is truncated just before the apex. The retro-optic is located behind the truncated pyramid in order to reflect the 1.2 $\mathrm{cm} \times 1.2 \mathrm{~cm}$ area at the center of the incident laser beam. An aluminum structure supports the four $45^{\circ}$ mirrors as well as the retro-optic to form a monolithic trap structure. The overall assembly is approximately $20 \mathrm{~cm}$ in diameter. The circularly polarized trapping and cooling laser is incident along the longitudinal $(z)$ axis of the pyramidal trap. This beam is retroreflected following two right-angle reflections from the pyramidal mirrors; each reflection also reverses the helicity of the circularly polarized beam so that the desired $\sigma^{+}-\sigma^{-}$polarization configuration is produced along all three orthogonal axes of the trap. A pair of anti-Helmholtz coils, oriented along the trap's longitudinal axis, provides the threedimensional quadrupole magnetic field for the magnetooptical trap. A schematic of the pyramidal trap is shown in Fig. 1.

The extraction column for atoms is defined by the 1-mm-diameter laser-drilled hole at the center of the retro-optic. This design approach enables us to construct the highest-quality hole for the small exit aperture in order to minimize scattered light from the edges and to achieve efficient extraction of atoms into the beam. The small aperture size was chosen to optimize the performance of this atom funnel as a continuous beam source while maintaining a low conductance $(C \approx 0.05 \mathrm{\ell} / \mathrm{s})$ between the vapor cell and the diagnostic region for differential pumping. A counter-propagating laser beam with the same detuning and intensity as the main trapping beam is optionally provided to fill in the extraction column. The pyramidal trap can be operated simply as a $3 \mathrm{D}-\mathrm{MOT}$ when this "fill" beam is present, and the coldatom beam source can also be operated in pulsed mode by switching the fill beam off and on with a mechanical shutter.

The trapping and cooling lasers consist of a Ti:sapphire ring laser, which provides up to $1 \mathrm{~W}$ of power at $852 \mathrm{~nm}$, and a $150-\mathrm{mW}$ DBR (distributed Bragg reflector) diode laser (SDL 5722). The Ti:sapphire laser serves as the main trapping laser and is detuned $5-30 \mathrm{MHz}$ to the red from the $6 S_{1 / 2}, F=4 \rightarrow 6 P_{3 / 2}, F^{\prime}=5$ transition in cesium.

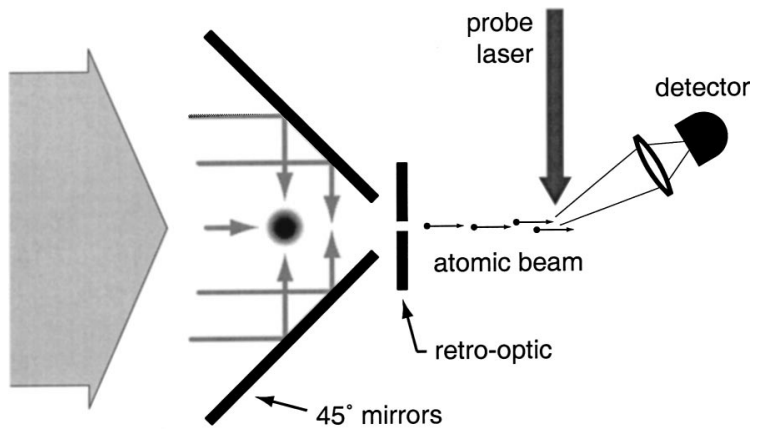

Fig. 1. Schematic diagram of the experimental apparatus and detection optics. The diameter of the pyramidal mirror assembly is approximately $20 \mathrm{~cm}$.
A second laser frequency is needed to depopulate the other hyperfine level of the ground state. This hyperfine repumping frequency is generated by the DBR laser locked to the $6 S_{1 / 2}, F=3 \rightarrow 6 P_{3 / 2}, F^{\prime}=4$ transition. The trapping and repump lasers are overlapped on a polarizing cube beam splitter, then expanded a factor of 30 by a telescope consisting of a short-focal-length lens plus a 25-cm-diameter gold-coated parabolic mirror $(f=1.5 \mathrm{~m})$. The parabolic mirror is used at close to normal incidence $\left(\theta_{i}<10^{\circ}\right)$ in order to minimize paraxial aberrations. The resulting collimated beam is approximately $15 \mathrm{~cm}$ in diameter, with a typical trapping laser intensity of $3 \mathrm{~mW} / \mathrm{cm}^{2}$. This beam enters the vapor cell via a 10 -in $(25-\mathrm{cm})$ conflat viewport.

The cesium vapor pressure in the source/collection region is varied between approximately $10^{-9}$ Torr and $10^{-7}$ Torr by heating a cesium reservoir connected to the vapor cell. The background cesium pressure is monitored with a resonant probe laser. The diagnostic region downstream of the aperture consists of a $32 \mathrm{~mm} \times 32$ $\mathrm{mm} \times 360 \mathrm{~mm}$ rectangular glass cuvette. The flat faces allow for excellent optical access for probe lasers and the fluorescence-imaging optics. A calibrated CCD camera with an $f / 6$ lens system is used for fluorescence imaging of the beam. A silicon photodiode is also used for fluorescence detection.

\section{BEAM CHARACTERIZATION}

The pyramidal trap was initially operated as a standard MOT by plugging the extraction column with a counterpropagating "fill" beam through the aperture. With the fill beam present, atoms in the pyramidal MOT form an ellipsoidal cloud with length of approximately $1.2 \mathrm{~cm}$ along the major axis. The size of this cloud decreases significantly as the fill beam is blocked and atoms are extracted from the trap toward the aperture. The slow beam of cold atoms in the extraction column are readily visible in fluorescence images of the pyramidal MOT (Fig. 2).

The cold-atom beam is monitored in the region downstream of the aperture by fluorescence detection. The glass cuvette allows viewing of the atomic beam at distances from $17 \mathrm{~cm}$ to over $50 \mathrm{~cm}$ downstream of the aperture. An external-cavity diode laser, locked to the $4 \rightarrow 5^{\prime}$ transition, provides the main probe beam. This beam is delivered through a polarizing optical fiber and collimated to give a 9.4-mm-diameter vertically polarized beam with 5-mW typical power. A few-milliwatt repump beam, tuned to the $3 \rightarrow 4^{\prime}$ transition, overlaps the main probe laser. We use lock-in detection to enhance our sensitivity in fluorescence detection of the atomic beam.

A calibrated CCD camera system (Cohu 4990 series camera plus $f / 6$ collection optics) is also available for fluorescence imaging of the cold-atom beam. The imaging camera allows the characterization of the spatial profile of the beam and is also used to measure the atom flux.

We discovered that the beam intensity was significantly increased with the introduction of an optical mask in front of the entrance window to reduce scattering from 


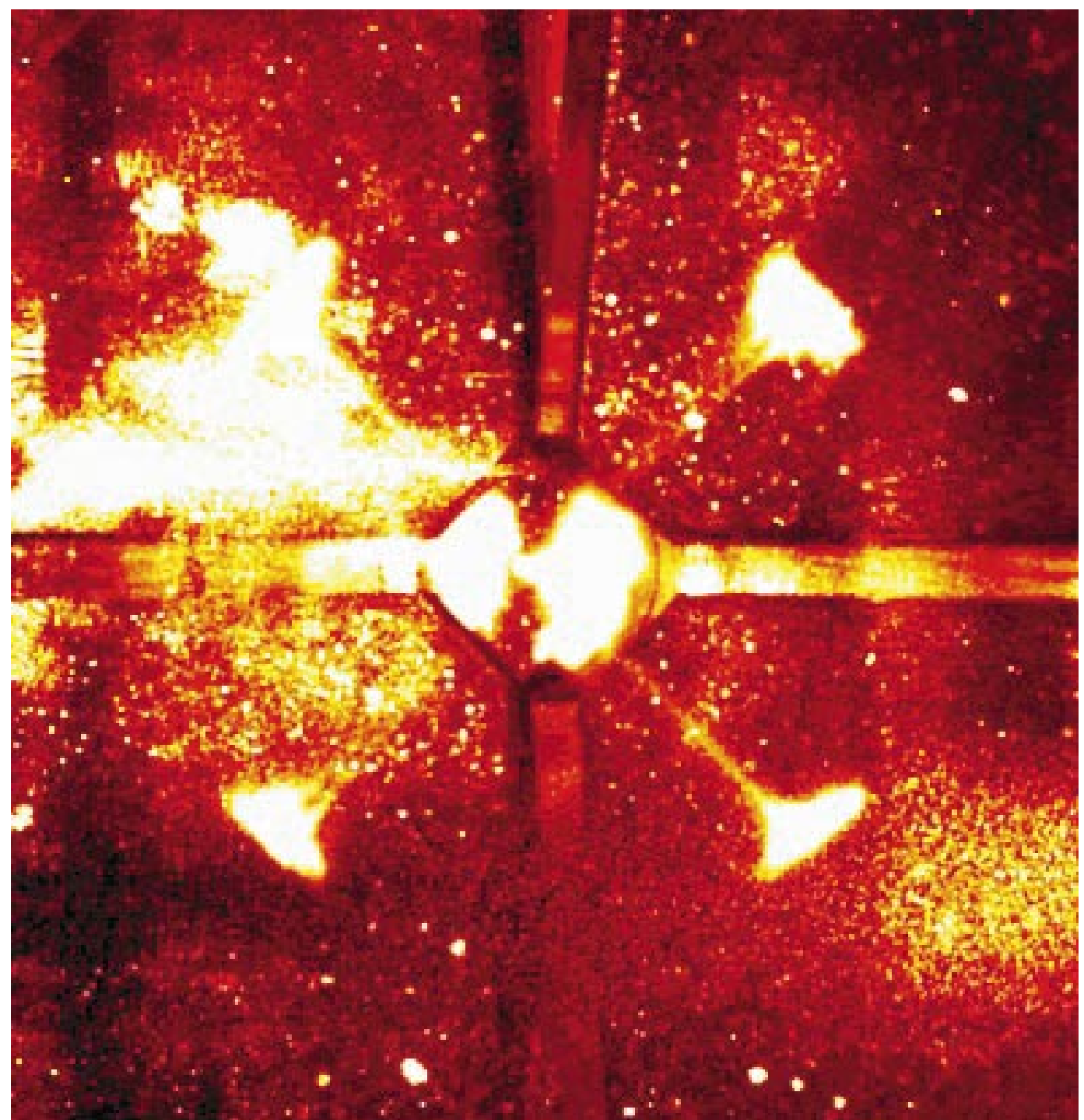

Fig. 2. False-color CCD image of fluorescence from trapped cesium atoms in the pyramidal magneto-optical trap. The atoms in the extraction column for the cold beam are visible in the side views afforded by the 45-deg mirrors.

the edges of the truncated pyramidal mirrors. The central region of the mask consists of a square "ring" of a size corresponding to the $1.2 \mathrm{~cm} \times 1.2 \mathrm{~cm}$ edges of the truncated pyramidal mirrors, and this square ring is supported by four narrow spokes extending radially outward to a 25 -cm-diameter outer ring. The radial spokes were aligned with the corner vertices of the pyramidal mirror assembly so as to not perturb the laser field at the center of the trap. The effect of this mask will be discussed more fully later.

\section{A. Mean Velocity}

The longitudinal velocity of the atomic beam is determined from the Doppler shift of the fluorescence spectrum resulting from excitation of the cold atoms by a probe laser incident at an angle $\phi$ with respect to the beam. We measure this Doppler shift $\Delta \nu_{\mathrm{D}}$ as a relative frequency shift between the laser-induced fluorescence spectra from this probe and from a reference probe oriented perpendicular to the beam. Both probes intersect the atomic beam at the same position $z^{\prime}$, so the Zeeman shifts identically cancel. The mean longitudinal velocity is then given by

$$
\left\langle v_{z}\right\rangle=\Delta \nu_{\mathrm{D}} \lambda / \cos \phi
$$

where $\lambda$ is the probe laser wavelength. The mean velocity measured by this technique is $15 \mathrm{~m} / \mathrm{s}$ for typical operating conditions.

\section{B. Atom Flux}

The calibrated CCD camera system allows the determination of the total (integrated) flux in the atomic beam from fluorescence images of the beam. For a cold-atom beam traveling with mean velocity $\left\langle v_{z}\right\rangle$ through a resonant probe laser with diameter $\Delta z$, the integrated atom flux (or particle current) $\mathfrak{I}$ is given by

$$
\mathfrak{I}=\frac{n_{\mathrm{c}}}{\eta} \frac{4 \pi}{d \Omega} \frac{\left\langle v_{z}\right\rangle}{\Delta z}\left(\frac{\Gamma}{2} \frac{S_{0}}{1+S_{0}}\right)^{-1},
$$


where $n_{\mathrm{c}}$ is the number of counts recorded by the imaging system with detector efficiency $\eta$ and collection solid angle $d \Omega, S_{0} \equiv I / I_{\text {sat }}$ is the saturation parameter for the probe laser, and $\Gamma$ is the natural linewidth of the atomic transition. Using Eq. (2), we measure a continuous beam flux of $\mathfrak{I}=2.2 \times 10^{9}$ atoms $/ \mathrm{s}$ at $15 \mathrm{~m} / \mathrm{s}$ for typical operating conditions.

\section{Beam Profile and Divergence}

The CCD camera system allows the determination of the spatial profile and divergence for the cold-atom beam. By translating the probe beam together with the camera system, we are able to obtain fluorescence images of the cold-atom beam over a $30-\mathrm{cm}$ range of positions along the beam axis $z$ in the cuvette cell. Off-resonant "dark" backgrounds are subtracted from the images to remove scattering from the uncoated windows of the cuvette. ${ }^{14}$ The transverse spatial profiles for the cold-atom beam are determined by deconvolving the line-of-sight integration from the fluorescence images, and the resulting profiles are fit to a Gaussian function to characterize the widths. The typical width for the beam is $3.6 \mathrm{~mm}$ (FWHM) measured $17 \mathrm{~cm}$ from the aperture.

The beam divergence is calculated directly by measuring the beam widths at several locations along the $z$ axis. The measured divergence angle $\vartheta$ is less than $16 \mathrm{mrad}$ (full angle) over a broad range of operating conditions. The divergence, though, is strongly dependent on laser detuning; this dependence is discussed in Section 5.

\section{SIMULATION DETAILS}

A semiclassical numerical model was developed by using MatLab $^{15}$ to simulate the behavior of atoms in the pyramidal trap-based beam source. The model calculates the scattering forces on the individual atoms as they interact with the optical and magnetic fields. The atoms are represented as two-level systems and induced dipole forces are ignored. Recoil heating, collisional losses, and the density-limiting forces due to multiple scattering are all incorporated into the model. This simulation approach allows the calculation of relative loading rates and numbers of trapped atoms for arbitrary trap geometries and sizes, as well as the formation and detailed dynamics of the cold-atom beam.

Starting with a Monte Carlo generated sample of atoms (up to $5 \times 10^{5}$ ) with initial conditions described by a truncated thermal distribution, ${ }^{16}$ we compute the equation of motion for each atom,

$$
\mathrm{d} \mathbf{p}=\sum_{i=1}^{N} \mathbf{F}_{i} \mathrm{~d} t+\mathrm{d} \mathbf{p}_{\text {recoil }}
$$

where $\mathbf{F}_{i}$ are the spontaneous scattering forces from each of the $N$ laser beams incident (either directly or by reflection from the trap mirrors) on the atom ${ }^{17}$ :

$$
\mathbf{F}_{i}=\hbar \mathbf{k}_{i} \frac{\Gamma}{2} \frac{S_{0}}{1+N S_{0}+4\left(\delta-\mathbf{k}_{i} \cdot \mathbf{v}+\mu^{\prime} B / \hbar\right)^{2} / \Gamma^{2}},
$$

where $\delta$ is the laser detuning from resonance $\omega_{\mathrm{L}}-\omega_{0}$, $\Gamma / 2 \pi=5.2 \mathrm{MHz}$ is the natural linewidth of the lasercooling transition, $\mu^{\prime} \equiv\left(g_{\mathrm{e}} M_{\mathrm{e}}-g_{\mathrm{g}} M_{\mathrm{g}}\right) \mu_{\mathrm{B}}$ is the effective magnetic moment, and $S_{0}$ is the saturation parameter for the trapping laser. All atom-laser interactions occur with a narrow single-frequency laser within a $10-\mu$ s time step, but at each time step the specific laser frequency $\omega_{\mathrm{L}}$ is selected from a Lorentzian-distributed series of frequencies (centered around the nominal value) generated by the Monte Carlo technique to approximate our $2-\mathrm{MHz}$ laser linewidth. Recoil heating ${ }^{18}$ is incorporated as the momentum increase

$$
\mathrm{d} \mathbf{p}_{\text {recoil }}=\sqrt{N_{s}} \hbar k \hat{\mathbf{e}}
$$

where $N_{s}$ is the total number of absorption-emission cycles during each time interval, and the recoil direction $\hat{\mathbf{e}}$ is chosen randomly from an isotropic distribution at each time step.

The current model also considers collective effects resulting from the large number of atoms in this lasercooled system. Radiation trapping within the optically dense trapped atom cloud is incorporated as a densitydependent repulsive force. ${ }^{19}$ Collisional losses from the trap are currently neglected since this loss rate is much smaller than the rate that atoms are extracted into the beam, but losses from the beam due to collisions with background cesium atoms are accounted for by modifying the calculated flux $\mathfrak{I}_{0}$ by an exponential loss factor

$$
\mathfrak{I}=\mathfrak{I}_{0} \exp \left[-\Gamma_{\text {coll }}\left\langle t_{\mathrm{e}}\right\rangle\right],
$$

where $\Gamma_{\text {coll }}=\left(n_{\mathrm{Cs}} \sigma_{\text {eff }}\left\langle v_{\text {rel }}\right\rangle\right)^{-1}$ is the collisional loss rate and $\left\langle t_{\mathrm{e}}\right\rangle$ is the mean time that atoms spend in the beam. The large value for the effective cross section, $\sigma_{\text {eff }}=0.6$ $\times 10^{-12} \mathrm{~cm}^{2},{ }^{20}$ reflects the dominant contribution from

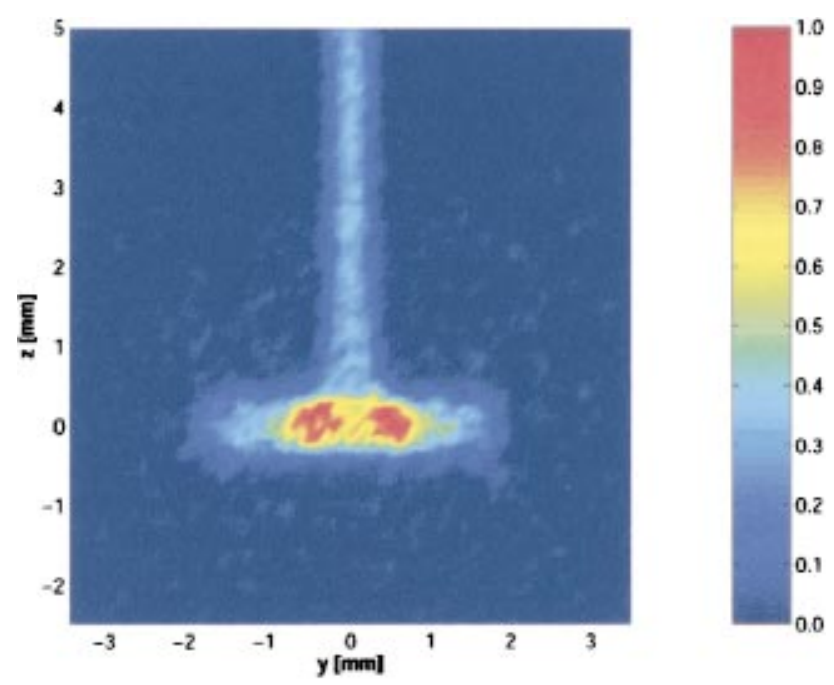

Fig. 3. Two-dimensional density plot illustrating the steadystate solution for the numerical model of the atomic-beam source based on the pyramidal trap. The total number of atoms in the simulation is $5 \times 10^{5}$. 
light-assisted collisions within the collection region. The detailed pressure dependence was not investigated in the current study, so this value for the cross section could not be verified. We note, however, that for the low pressures in the current experiment, the mean time between collisions $1 / \Gamma_{\text {coll }}$ is much longer than the characteristic escape time $\left\langle t_{\mathrm{e}}\right\rangle$; thus the effect of collisional losses on the atomic beam is minimal.

We have verified that this model gives the expected size and density for a conventional 3D-MOT, and it returns equilibrium temperatures consistent with the Dopplercooling limit for both two- and three-dimensional optical molasses. The current experiment was described by modifying the Gaussian beam profiles for the trapping and repumping lasers to account for our pyramidal trap geometry. With these modifications, the model predicts a maximum capture velocity of $29 \mathrm{~m} / \mathrm{s}$ along the longitudinal axis of our trap.

To simulate the radiation imbalance created by the aperture in the retro-optic, the laser beam propagating along the $-z$ axis is "turned off" within the narrow cylindrical volume of the extraction column so that atoms within this region interact only with the laser fields along the other five axes. Once the extraction column is created, the cold-atom beam flux reaches a steady-state value within 3-11 ms. The steady-state solution is illustrated in Fig. 3, where the two-dimensional density plot clearly reveals both the trapped atoms and the continuous beam of cold atoms extracted from the trap. This plot reproduces the essential features of the experimentally observed fluorescence images (compare with reflected side view images in Fig. 2), although we note that, in the fluorescence images, the extracted beam becomes increasingly faint away from the trap center as the cold atoms are shifted further out of resonance by the combined Doppler and Zeeman shifts.

Calculated results for mean velocity, divergence, and integrated flux are presented in the following section.

\section{RESULTS AND DISCUSSION}

We measured a continuous atom flux of 2.2 $\times 10^{9}$ atoms $/ \mathrm{s}$ at $15 \mathrm{~m} / \mathrm{s}$ from our cold-atom beam source. The divergence of this beam was $15 \mathrm{mrad}$, and the corresponding radiant intensity was $1.2 \times 10^{13}$ atoms $s^{-1} \mathrm{sr}^{-1}$. We also measured instantaneous peak values of $4 \times 10^{9}$ atoms/s by chopping the repump laser in the collection region. It is not clear whether this enhancement was due to complicated dynamical effects involving the loading of the MOT or due to the depletion of a significant portion of the low-velocity cesium atoms from the background vapor. The latter case is more likely if the surfaces in the vacuum chamber were not completely passivated with cesium, in which case the rethermalization time $\tau$ for the cesium vapor can be long compared with $1 / \Gamma_{\text {beam }}$, where $\Gamma_{\text {beam }}$ is the rate atoms are extracted from the trap. This case suggests that the steady-state performance of the cold-atom beam source was limited by the low pressure $\left(3 \times 10^{-9}\right.$ Torr $)$ at the time of this measurement, although this behavior was not investigated further.

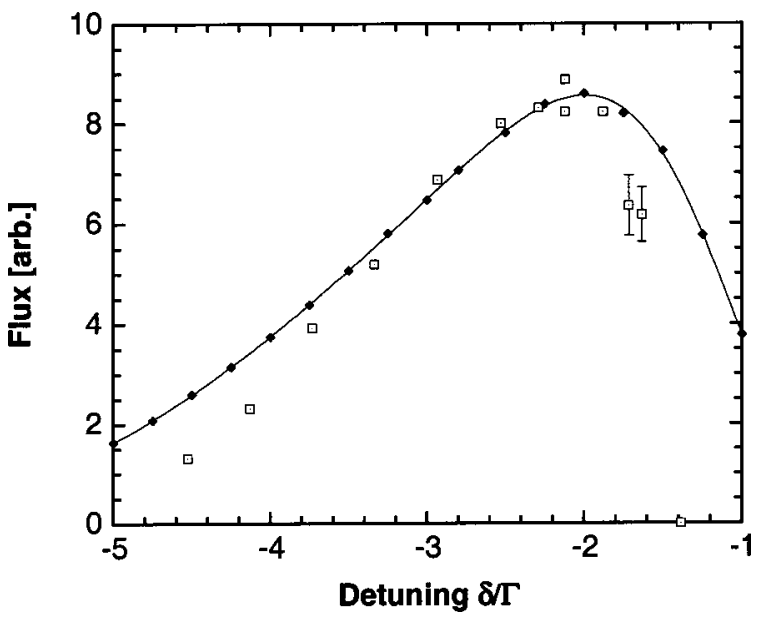

Fig. 4. Dependence of atomic-beam flux on laser detuning. Simulation results, indicated by the solid diamonds, have been scaled to provide a best fit near the peak. The smooth curve is provided only to guide the eye.

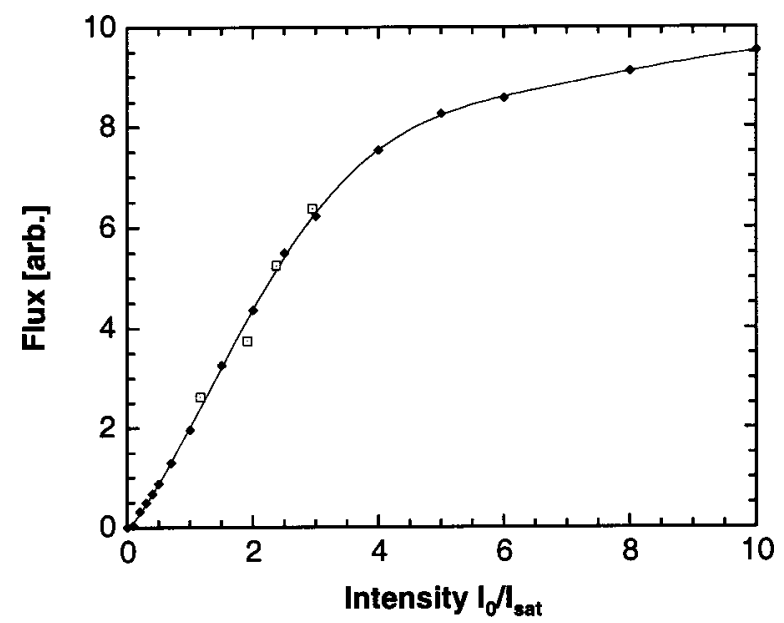

Fig. 5. Atom flux versus laser intensity. Simulation results, indicated by the solid diamonds, have been scaled to provide a best fit with experimental results, and the smooth curve is provided to guide the eye.

The integrated flux was observed to decrease by at least an order of magnitude when the input mask is removed or deliberately misaligned, indicating that laser light scattered from the truncated edges of the pyramidal mirror assembly results in significant losses of atoms from the slow beam. We believe that, with increased care in the manufacture of the mirror assembly, this scattering can be greatly reduced and the need for this mask eliminated. The flux measured with the mask in place, however, is still less than one might predict ${ }^{21,22}$ for the very large trap volume in the present experiment. We have investigated the scaling properties of pyramidal and conventional MOTs using our numerical model, and these simulations indicate that the loading rate increases more slowly with increasing trap volume in the pyramidal MOT than in a conventional six-beam MOT. Using scaling arguments based on these results, one would predict an atom flux greater than $10^{10}$ atoms/s from our large $\left(\sim 300-\mathrm{cm}^{3}\right)$ trap. The discrepancy between the predicted and observed values may indicate that additional losses are present within the beam. We note that for the pyra- 
midal trap geometry in particular, atoms within the long extraction column are subject to scattering losses due to asymmetries in the transverse spatial profile of the trapping laser; therefore the atomic-beam performance may further improve with spatial filtering of the laser beam.

Maximum values for the atom flux were observed at a relatively small laser detuning $(\delta=-2.1 \Gamma)$ as compared with the reported optimal values for cold-atom beams derived from conventional MOTs $\left(-3.3 \Gamma,{ }^{6}-5 \Gamma^{8}\right)$.

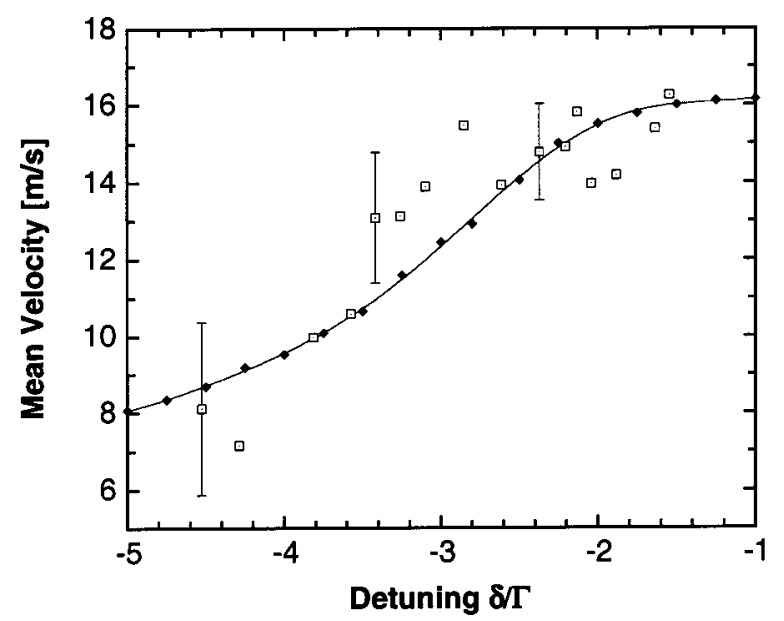

Fig. 6. Mean longitudinal velocity versus laser detuning. Simulation results are indicated by the solid diamonds, and the smooth curve is provided to guide the eye. Error bars on selected experimental points are representative of the uncertainty associated with our analysis method.

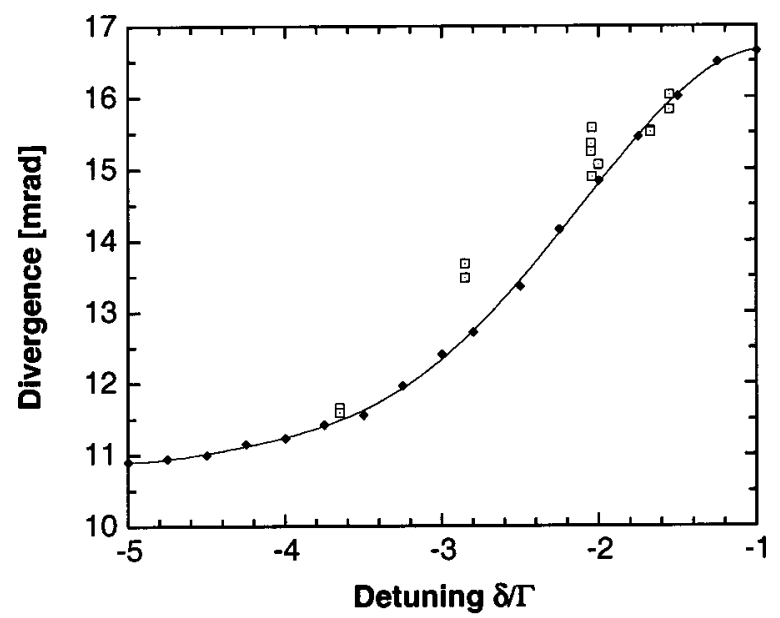

Fig. 7. Atomic-beam divergence versus laser detuning. Simulation results are indicated by the solid diamonds, with the smooth curve intended to guide the eye.
Our detuning, however, is comparable to the reported value $(-2.2 \Gamma)$ that maximizes the number of atoms in the pyramidal trap. ${ }^{10}$ The measured flux decreased rapidly as the trapping laser was tuned in either direction from the optimal detuning frequency, as illustrated in Fig. 4. Figure 4 also presents results from our numerical model. The Monte Carlo simulations consider only a finite number of atoms $\left(N \sim 10^{5}\right)$ within a closed system, so only relative values for the atom flux were obtained. Nevertheless, the model confirms the optimal laser-detuning frequency and reproduces the overall dependence on detuning, although the calculated flux varies less rapidly than the measured values as the laser frequency is varied from this optimal detuning. We found the incorporation of a finite laser linewidth had little or no effect on the calculated flux over this range of laser detunings.

The dependence of atom flux on laser intensity was also investigated. Experimental results suggest a near-linear dependence of flux on laser intensity for lower intensities, but simulation results predict a saturation effect at higher intensities, i.e., $S_{0} \gtrsim 4$ (see Fig. 5). The range of intensities investigated experimentally was limited by the available laser power $(600 \mathrm{~mW})$ at the time of the measurement. We note, however, that our model predicts that the flux should increase by, at best, one-third with a factor of 2 increase in laser power.

The mean longitudinal velocity $\left\langle v_{z}\right\rangle$ was $15 \mathrm{~m} / \mathrm{s}$ for typical operating conditions. This velocity was approximately constant for laser detunings less than $-3 \Gamma$. Beyond this value, $\left\langle v_{z}\right\rangle$ decreased with increasing (i.e., more negative) trap laser detunings, as illustrated in Fig. 6. The longitudinal velocities calculated by our numerical model were in quantitative agreement with measured values over the range of laser detunings investigated experimentally. This model indicates that the acceleration due to scattering forces and the resulting terminal velocities of atoms in the beam are effectively limited by the attenuation of the "push" laser beam by the optically dense trapped cloud; agreement between calculated and measured velocities at small detunings was degraded when these attenuation effects were neglected.

The divergence $\vartheta$ of the cold-atom beam was $15 \mathrm{mrad}$ (FWHM) for the laser detuning that optimizes the atom flux, corresponding to a transverse temperature of 200 $\mu \mathrm{K}$. The measured divergence angle was strongly dependent on laser detuning, however, as shown in Fig. 7. At the largest laser detuning at which the divergence could be measured $(-3.65 \Gamma)$, this angle had decreased to 11.5 mrad, corresponding to a transverse temperature of 54 $\mu \mathrm{K}$.

Table 1. Summary of Atomic-Beam Performance in the Present Work, and Comparison with Reported Values from Selected Slow $\left(\left\langle v_{z}\right\rangle<30 \mathrm{~m} / \mathrm{s}\right)$ Atomic-Beam Sources

\begin{tabular}{|c|c|c|c|c|c|}
\hline Parameter & Present Work & Lu et al. ${ }^{a}$ & Dieckmann et al. ${ }^{b}$ & Arlt et al. ${ }^{c}$ & Camposeo et al. ${ }^{d}$ \\
\hline Radiant intensity [atom $\mathrm{s}^{-1} \mathrm{sr}^{-1}$ ] & $1.2 \times 10^{13}$ & $5 \times 10^{12}$ & $6.2 \times 10^{12}$ & $8.5 \times 10^{11}$ & $7.5 \times 10^{12}$ \\
\hline Flux $\mathfrak{I}, \mathrm{cw}[$ atom $/ \mathrm{s}]$ & $2.2 \times 10^{9}$ & $5 \times 10^{9}$ & $9 \times 10^{9}$ & $1.1 \times 10^{9}$ & $4 \times 10^{9}$ \\
\hline Divergence $\vartheta$, FWHM [mrad] & $11-16$ & $5-36$ & 43 & 40 & $26 \pm 1$ \\
\hline Mean velocity $\left\langle v_{z}\right\rangle[\mathrm{m} / \mathrm{s}]$ & $7-16$ & 14 & 8 & $8-12$ & $8-12$ \\
\hline
\end{tabular}

${ }^{a}$ Low-velocity intense source (3D-MOT), Ref. 8.

${ }^{b} 2 \mathrm{D}^{+}$-MOT, Ref. 6 .

${ }^{c}$ Pyramidal funnel, Ref. 12.

${ }^{d}$ Pyramidal funnel, Ref. 13. 
The divergence calculated by the model reproduces this dependence, even without the incorporation of a subDoppler cooling mechanism. This close agreement is explained upon examination of the individual atom trajectories during a simulation, which reveals that the divergence of the atomic beam is primarily determined by a geometric collimation mechanism. The geometrically limited divergence angle can be approximated as $\vartheta_{\text {geom }}$ $=D /\left(\left\langle t_{\mathrm{e}}\right\rangle\left\langle v_{z}\right\rangle\right)$, where $\left\langle t_{\mathrm{e}}\right\rangle$ is the mean transit time for atoms traveling from the center of the trap (where atoms enter this atom funnel) to the exit aperture, and $D$ is the aperture diameter. The decreasing divergence angle reported by the numerical model results from the increasing transit times at larger laser detunings. At the detuning of $-5 \Gamma$, for example, the mean transit time is calculated to be $11 \mathrm{~ms}$, giving a geometrically limited divergence of $11 \mathrm{mrad}$. We note that atoms with hotter transverse temperatures are not lost completely once they leave the extraction column but are efficiently recycled back to the center of the trap where they re-enter the atom funnel, as demonstrated in our simulations. The inclusion of sub-Doppler cooling in our model may reduce the average number of times an individual atom is "recycled" before exiting through the aperture but is not necessary to reproduce the observed features of the experiment.

A comparison of the experimental measurements to previously reported values is provided in Table 1 .

\section{SUMMARY AND CONCLUSIONS}

We have demonstrated an intense source for a slow beam of cold cesium atoms based on a modified pyramidal magneto-optical trap. An integrated flux of 2.2 $\times 10^{9}$ atoms/s was obtained in a continuous atomic beam with a mean velocity of $15 \mathrm{~m} / \mathrm{s}$ and divergence of $15 \mathrm{mrad}$, and the corresponding radiant intensity was 1.2 $\times 10^{13}$ atoms s${ }^{-1} \mathrm{sr}^{-1}$. This radiant intensity is believed to be the highest value reported for any slow atom beam to date. The low mean velocity and small divergence of our cold-atom beam should facilitate loading of an ultrahigh vacuum MOT with near-unity capture efficiency.

The performance of our beam source was characterized over a range of operating conditions, and measured values for flux, mean velocity, and divergence for the coldatom beam were in good agreement with results from Monte Carlo numerical simulations. The numerical model developed here is equally capable of describing laser-cooled systems with different trap geometries, as well as multiple trap systems. This model is currently being employed as a design tool in the development of compact high-performance cold-atom beam sources for future NASA microgravity flight projects.

We have also recently demonstrated a dual-species (Cs $+\mathrm{Rb}$ ) beam in this apparatus using four laser frequencies for trapping and cooling. ${ }^{23}$ The cesium and rubidium beams can be operated simultaneously or individually, and we have measured comparable values for the flux in each species beam. The slow rubidium beam has been used to directly load a second MOT oper- ating downstream at pressures of approximately $10^{-11}$ Torr. Details of the dual-species system will be reported in the future.

\section{ACKNOWLEDGMENTS}

This research was carried out at the Jet Propulsion Laboratory, California Institute of Technology, under a contract with the National Aeronautics and Space Administration, and was supported by the Jet Propulsion Laboratory Director's Research and Development Fund. J. M. Kohel also thanks the National Research Council for support during this work. Thanks are due to N. Guise, B. Klipstein, D. Seidel, B. Young, and N. Yu for valuable technical support and discussions.

\section{REFERENCES AND NOTES}

1. W. D. Phillips and H. Metcalf, "Laser deceleration of an atomic beam," Phys. Rev. Lett. 48, 596-599 (1982).

2. W. Ertmer, R. Blatt, J. L. Hall, and M. Zhu, "Laser manipulation of atomic beam velocities: demonstration of stopped atoms and velocity reversal," Phys. Rev. Lett. 54, 996-999 (1985).

3. S. Weyers, E. Aucouturier, C. Valetin, and P. Dimarcq, "A continuous beam of cold cesium atoms extracted from a two-dimensional magneto-optical trap," Opt. Commun. 143, 30-34 (1997).

4. P. Berthoud, A. Joyet, G. Dudle, N. Sagna, and P. Thomann, "A continuous beam of slow, cold cesium atoms magnetically extracted from a 2D magneto-optical trap," Europhys. Lett. 41, 141-146 (1998).

5. P. Berthoud, E. Fretel, and P. Thomann, "Bright, slow, and continuous beam of laser-cooled cesium atoms," Phys. Rev. A 60, R4241-R4244 (1999).

6. K. Dieckmann, R. J. C. Spreeuw, M. Weidemüller, and J. T. M. Walraven, "Two-dimensional magneto-optical trap as a source of slow atoms," Phys. Rev. A 58, 3891-3895 (1998).

7. H. Chen and E. Riis, "Cold atomic beam from a rubidium funnel,” Appl. Phys. B 70, 665-670 (2000).

8. Z. T. Lu, K. L. Corwin, M. J. Renn, M. H. Anderson, E. A. Cornell, and C. E. Wieman, "Low-velocity intense source of atoms from a magneto-optical trap," Phys. Rev. Lett. 77, 3331-3334 (1996).

9. This quantity (atom s $\mathrm{s}^{-1} \mathrm{sr}^{-1}$ ) is sometimes referred to as the "brightness" of an atomic beam, although brightness more commonly refers to the radiometric quantity of radiance, or radiant intensity per unit of projected area.

10. K. I. Lee, J. A. Kim, H. R. Noh, and W. Jhe, "Single beam atom trap in a pyramidal and conical hollow mirror," Opt. Lett. 21, 1177-1179 (1996).

11. R. S. Williamson III, P. A. Voytas, R. T. Newell, and T. Walker, "A magneto-optical trap loaded from a pyramidal funnel," Opt. Express 3, 111-117 (1998).

12. J. J. Arlt, O. Maragò, S. Webster, S. Hopkins, and C. J. Foot, "A pyramidal magneto-optical trap as a source of slow atoms," Opt. Commun. 157, 303-309 (1998).

13. A. Camposeo, A. Piombini, F. Cervelli, F. Tantussi, F. Fuso, and E. Arimondo, "A cold cesium atomic beam produced out of a pyramidal funnel,” Opt. Commun. 200, 231-239 (2001).

14. Cesium background pressure was initially negligible in the probe region. Later, as the vapor pressure increased, background images were obtained with the probe laser onresonance and the atomic beam interrupted by blocking the repump laser in the MOT.

15. MathWorks, Inc., 3 Apple Hill Drive, Natick, MA 017602098. 
16. The Maxwell-Boltzmann velocity distribution is modified so that $f\left(v>v_{c}\right)=0$, where $v_{c}$ is the maximum capture velocity of the pyramidal MOT

17. See, for example, H. J. Metcalf and P. van der Straten, $L a$ ser Cooling and Trapping (Springer-Verlag, New York, 1999).

18. P. D. Lett, W. D. Phillips, S. L. Rolston, C. E. Tanner, R. N. Watts, and C. I. Westbrook, "Optical molasses," J. Opt. Soc. Am. B 6, 2084-2107 (1989).

19. D. W. Sesko, T. G. Walker, and C. E. Wieman, "Behavior of neutral atoms in a spontaneous force trap," J. Opt. Soc. Am. B 8, 946-958 (1991).

20. A. M. Steane, M. Chowdhury, and C. J. Foot, "Radiation force in the magneto-optical trap,” J. Opt. Soc. Am. B 9 $2142-2158$ (1992).

21. K. Gibble, S. Kapasi, and S. Chu, "Improved magnetooptical trapping in a vapor cell," Opt. Lett. 17, 526-528 (1992).

22. K. Lindquist, M. Stephens, and C. Wieman, "Experimental and theoretical study of the vapor-cell Zeeman optical trap," Phys. Rev. A 46, 4082-4090 (1992).

23. N. Lundblad, D. Aveline, R. J. Thompson, W. Klipstein, J. Kohel, J. Ramirez-Serrano, D. Enzer, and L. Maleki, "Production and characterization of a dual-species cold atomic beam,” Bull. Am. Phys. Soc. 47, 21 (2002). 